\title{
Separation of natural colorants from the fermented broth of filamentous fungi using colloidal gas aphrons
}

\author{
Valéria Carvalho Santos-Ebinuma ${ }^{\mathrm{a}, *}$, Maria Francisca Simas Teixeira ${ }^{\mathrm{b}}$, Adalberto Pessoa Jr. , $^{\text {, }}$ \\ Paula Jauregi ${ }^{\mathrm{d}}$ \\ ${ }^{a}$ Department of Bioprocess and Biotechnology, School of Pharmaceutical Sciences, UNESP-Univ. Estadual Paulista, Araraquara, SP, Brazil \\ ${ }^{\mathrm{b}}$ Department of Biochemical and Pharmaceutical Technology, University of São Paulo, Avenida Prof. Lineu Prestes 580, Bl. 16, 05508-900 São Paulo, SP, Brazil \\ ${ }^{c}$ Culture Collection DPUA/UFAM, Universidade Federal do Amazonas, Av. Gal. Rodrigo Octávio Jordão Ramos, 3000, 69.077-000 Manaus, AM, Brazil \\ ${ }^{\mathrm{d}}$ Department of Food and Nutritional Sciences, The University of Reading, Whiteknights, Reading RG6 6AP, UK
}

\section{A R T I C L E I N F O}

\section{Article history:}

Received 30 September 2015

Received in revised form 15 February 2016

Accepted 22 February 2016

Available online 23 February 2016

\section{Keywords:}

Natural colorants

Bioprocess

Surfactants

Colloidal gas aphron

Extraction

\begin{abstract}
A B S T R A C T
There is a worldwide interest in the development of processes for producing colorants from natural sources. Microorganisms provide an alternative source of natural colorants produced by cultivation technology and extracted from the fermented broth. The aim of the present work was to study the recovery of red colorants from the fermented broth of Talaromyces amestolkiae using the technique of colloidal gas aphrons (CGA) comprising surfactant-stabilized microbubbles. Preliminary experiments were performed to evaluate the red colorants' solubility in different organic solvents, octanol/water partitioning, and their stability in surfactant solutions, namely hexadecyl trimethylammonium bromide (CTAB), sodium dodecyl sulfate (SDS), and polyoxyethylenesorbitan monolaurate (Tween 20 ), which are cationic, anionic and nonionic surfactants, respectively. The first recovery experiments were carried out using CGA generated by these surfactants at different volumetric ratios $\left(V_{R}, 3-18\right)$. Subsequently, two different approaches to generate CGA were investigated at $V_{R}$ values of 6 and 12: the first involved the use of CTAB at pH 6.9-10.0, and the second involved the use of Tween 20 using red colorants partially dissolved in ethanol and Tween 20 . The characterization results showed that red colorants have a hydrophilic nature. The highest recoveries were obtained with Tween 20 (78\%) and CTAB (70\%). These results demonstrated that the recovery of the colorants was driven by both electrostatic and hydrophobic interactions. The $V_{R}$ was found to be an important operating parameter and at $V_{R} 12$ with CTAB (at pH 9) maximum recovery, partitioning coefficient $(K=5.39)$ and selectivity in relation to protein and $\operatorname{sugar}\left(S_{P}=3.75\right.$ and $S_{S}=7.20$ respectively) were achieved. Furthermore, with Tween 20, the separation was driven mainly by hydrophobic interactions. Overall CGA show promise for the recovery of red colorants from a fermented broth. Although better results were obtained with CTAB than with Tween 20 the latter may be more suitable for some application due to its lower toxicity.
\end{abstract}

(c) 2016 Elsevier B.V. All rights reserved.

\section{Introduction}

Synthetic and natural colorants are extensively used in the food, cosmetic, and pharmaceutical industries [1,2]. However, natural colorants have recently gained popularity over synthetic ones, which, in some cases, are known to be potential carcinogens $[1,3]$. Therefore, there is a growing demand for ecofriendly/nontoxic colorants, specifically for the coloration of foods and

\footnotetext{
* Corresponding author at: Department of Bioprocess and Biotechnology, School of Pharmaceutical Sciences, UNESP-Univ. Estadual Paulista, Rodovia AraraquaraJaú/km 01, 14801-902 Araraquara, SP, Brazil.

E-mail address: valeriac@fcfar.unesp.br (V.C. Santos-Ebinuma).
}

pharmaceuticals, as well as for dyeing kids' cloths and leather garments $[4,5]$.

Natural colorants can be produced by microorganisms such as fungi species [6]. Fungal colorants show diversity not only in their chemical structures but also in the color range of their colorants, possibly adding new or additional hues to the color palettes of the existing colorants derived from contemporary sources $[7,8]$. Most of the colorants produced by fungi are quinones, flavonoids, melanins, and azaphilones, which belong to the aromatic polyketide chemical group and have been widely described for medicinal applications and potential use as dyes [9]. The structures of polyketides are known to contain loose d-electrons, as they often contain polyunsaturated functionality, i.e., ring systems, one or more 
carbonyl groups, carboxylic acid, and ester or amide functional groups exhibiting characteristic UV-vis spectra [8].

Monascus species is the most studied candidate for producing colorants [6]. However, alternative colorant-producing organisms are of great interest [2]. In this context, in the literature new producers of Monascus-like azaphilone colorants in liquid media such as Talaromyces amestolkiae (cited before as Penicillium purpurogenum) has been reported [6]. Furthermore, in studies performed by our research group, this microorganism showed the potential to produce natural colorants with significant antimicrobial activities, complete absence of toxicity against Artemia salina [10], and the ability to produce natural colorants stable under alkaline conditions, having good compatibility with polyethylene glycol (PEG) and sodium polyacrylate (NaPA) polymers to up to $70^{\circ} \mathrm{C}$ [11].

Most of the research on natural colorants derived from microorganisms deals with their production. However, the extraction and/ or purification of such colorants using new methods are of great interest, because most of the reported studies use organic solvents to extract the colorants from the fermented broth $[4,7,12,13]$. The use of organic solvents has some limitations, particularly in largescale applications, because of their high cost, toxicity of solvents, potential environmental impact, and because organic solvents may lead to irreversible product degradation [14]. Thus, the development of more efficient and cost-effective separation and purification processes is crucial to improve the process efficiency and economics, while maintaining the required high quality standards for market approval $[15,16]$.

In the search for alternative methods, there is a growing interest on the application of surfactants to separation processes [17]. Surfactants are amphiphilic molecules composed of a hydrophilic or polar moiety, known as the head, and a hydrophobic or nonpolar moiety, known as the tail. The surfactant head can be charged, dipolar (zwitterionic), or noncharged [18]. Sodium dodecyl sulfate (SDS), hexadecyltrimethylammonium bromide (CTAB), and polyoxyethylenesorbitan monolaurate (Tween 20) are examples of anionic, cationic, and nonionic surfactants, respectively.

Surfactants possess several unique characteristics such as the tendency to adsorb onto surfaces, to associate in solution to form micelles that dissolve nonpolar solutes [19,14], and the fact that most of them are nontoxic and biodegradable. Moreover, these systems usually have low energy requirements $[20,14]$.

One application of surfactants in the extraction process is their use with colloidal gas aphrons (CGA). CGA are surfactant-stabilized microbubbles $(10-100 \mu \mathrm{m})$ which can be generated by stirring a surfactant solution at high speeds $(8000 \mathrm{rpm})[21,22]$ or by sonication and homogenization [23]. The core of the microbubble is composed of a gas surrounded by a thick multilayer shell, which is formed of an inner surfactant film enveloped by a viscous aqueous layer [24]. The double layer of surfactants provides rigidity and low permeability to the structure while imparting some hydrophilic character to it [24].

CGA can be generated by ionic and nonionic surfactants, which will influence the outer surface of the microbubble to be positive, negative, or noncharged, respectively, to which oppositely or noncharged molecules will adsorb, resulting in their effective separation from the bulk liquid $[25,14]$. Thus, the adsorption selectivity of the surfactant can be adjusted [26,14]. CGA exhibit unique characteristics, including high interfacial area and high stability compared to those of conventional foams; they can also be pumped and separated easily from the liquid phase without any mechanical aid. Furthermore, the use of biodegradable surfactants results in environmentally friendly processes, and the final product can also be safe for human consumption [14]. These properties help to reduce the number of operations required in product purification/recovery, making CGA a cost-effective separation technique compared to conventional methods such as centrifugation and supercritical fluid extraction [27].

In light of their favorable properties, researchers have considered various applications of CGA, with a particular focus on the separation processes [27]. They have been successfully used to recover proteins from whey [28], polyphenols from wine waste extracts [25], carotenoids from plant extracts [22], astaxanthin particles from a fermentation mixture containing yeast cells [29], and pulp fibers from paper machine backwater [30].

As red colorants are one of the most important dyes used in many industries [3], the aim of this study was to investigate the recovery of red colorants from the fermented broth of $T$. amestolkiae using CGA generated by different surfactants under several conditions.

\section{Material and methods}

\subsection{Materials}

CTAB, SDS, Tween 20, octanol and bicinchoninic acid assay kit was obtained from Sigma Chemicals (St. Louis, MO). All the other reagents were of analytical grade and were used as received. The laboratory mixer (SL2T) fitted with four bladed impeller $(D=30 \mathrm{~mm})$ surrounded by a high shear screen and with a digital readout of the impeller speed was supplied by Silverson (Waterside, Bucks, UK). The spectrophotometers used were an Ultrospec 1100 pro purchased from Amersham Pharmacia Biotech (Biochrom, Cambridge, UK) and a PerkinElmer lambda 20 UV-vis Spectrophotometer coupled with a microcomputer. The $\mathrm{pH}$ was measured with a bench-top pH meter from Whatman (PHA 320, Kent, UK).

\subsection{Fermentation process}

T. amestolkiae was the microorganism used to produce the red colorants. It was kindly provided by the Culture Collection by Federal University of Amazon, DPUA, AM, Brazil. The cultures preserved in distilled water were reactivated in Czapeck Yeast Extract Agar (CYA) [31]. The cultures were maintained at $25^{\circ} \mathrm{C}$ for 7 days and after maintained in fridge at $4{ }^{\circ} \mathrm{C}$. The inoculum was prepared in CYA plate and the cultures were maintained at the same conditions of reactivation.

For fermentation, 5 mycelial agar discs ( $8 \mathrm{~mm}$ diameter) from inoculum were obtained by a self-designed cutter and transferred to $25 \mathrm{~mL}$ of fermentation medium in $125 \mathrm{~mL}$ Erlenmeyer flasks and incubated in orbital shaker incubator under $30^{\circ} \mathrm{C}, 150 \mathrm{rpm}$ for 15 days. The fermentation medium composition was liquid Czapeck Yeast Extract with modifications as described by SantosEbinuma et al. [11]. The media had the pH adjusted to 4.5 with $\mathrm{HCl}(5 \mathrm{M})$. After fermentation, the fermented broth was filtrated. The supernatant containing the red colorants was frozen in ultrafreezer at $-70^{\circ} \mathrm{C}$ to be used in the partitioning studies. All culture media prepared was autoclaved at $121{ }^{\circ} \mathrm{C}$ for $15 \mathrm{~min}$.

\subsection{Generation of CGA}

The SDS, Tween 20 and CTAB pH 6.9 solutions were prepared in deionised water. The CTAB pH 8.0 was prepared in Mcllvaine's buffer and the CTAB pH 9.0 and 10.0 solutions were prepared in Sodium Carbonate-bicarbonate Buffer. The SDS and CTAB solutions were prepared at concentration $2 \mathrm{mM}$ while the Tween 20 solution at concentration $20 \mathrm{mM}$. CGA were generated by stirring $300 \mathrm{~mL}$ of CTAB, SDS or Tween 20 solution, at $8000 \mathrm{rpm}$ for $5 \mathrm{~min}$ at room temperature using a high-speed impeller (Silverstone SL2T). 


\subsection{Characterization of CGA}

\subsubsection{Gas hold-up}

Gas hold-up $(\varepsilon)$ was determined following the method described by Amiri and Woodburn [32]. After generation, CGA were poured into a $1000 \mathrm{~mL}$ measuring cylinder and the volume of the clear liquid below the CGA dispersion was measured at 1 min intervals. After the dispersion collapse, the volume of the liquid was measured. This corresponded to the initial volume of the surfactant solution $\left(V_{10}\right)$ in the CGA dispersion [14]. The gas holdup $(\varepsilon)$, was defined as the ratio of gas volume $\left(V_{g}\right)$ to the dispersion volume $\left(V_{\mathrm{a} 0}\right)$. It was calculated according the Eq. (1) [14,27]:

$\varepsilon=\frac{V_{g}}{V_{a 0}}=\frac{V_{a 0}-V_{l 0}}{V_{a 0}} \times 100(\%)$

\subsubsection{Stability}

The stability of CGA was evaluated by measuring the half-life of the dispersion. The half-life $(\tau)$ was defined as the time required for half the initial volume of liquid to drain (Eq. (2)) [14,28].

$\tau=\frac{V_{10}}{2}$

\subsection{Red Colorants recovery from fermented broth using CGA}

The flotation column is made of Perspex glass $(0.04 \mathrm{~m}$ in internal diameter and $0.5 \mathrm{~m}$ in height). Initially, the fermented broth at absorbance of 2.00 Units of Absorbance ${ }_{490 \mathrm{~nm}}\left(\mathrm{UA}_{490 \mathrm{~nm}}\right.$ ) (the absorbance was standardized in all experiments to minimize the errors) was introduced into the column in the appropriate volume. CGA were generated as described in Section 2.4. Then, the speed was adjusted and maintained at $6000 \mathrm{rpm}$ to prevent creaming and avoid damaging of CGA structure [33]. A peristaltic pump (Watson and Marlow 502S) was used to introduce CGA into the column at different flow rates according to Eq. (3), changing in this way the contact time between the fermented broth and the surfactant. Once the column was filled, the mixture was left standing for 10 min before pumping out the separated liquid $\left(V_{\mathrm{LP}}\right)$ and aphron
$\left(V_{\mathrm{AP}}\right)$ phase. The volumes of separated liquid and aphron phase were measured. The experimental scheme is shown at Fig. 1.

Firstly, experiments were carried out at different surfactant/fermented broth volumetric ratio $\left(V_{R}\right.$, Eq. (3)) varying between 3 and 18 to the surfactants Tween 20, SDS and CTAB at pH 6.9. Secondly, the experiments were carried out in different ways at $V_{R}$ of 6 and 12. One set of experiments were done with CTAB at $\mathrm{pH}$ 6.9, 8.0, 9.0 and 10.0. The other experiments were performed with CGA generated by Tween 20 and the fermented broth partial dissolved at Ethanol (20:80 and 40:60) and Tween 20 (20:80, 40:60 and $60: 40$ ). The amount of sugar, proteins and red colorant were determined in each phase.

$V_{R}=\frac{V_{C G A}}{V_{F B}}=\frac{\text { Flow rate } \times \text { contact time }}{V_{F B}}$

The partitioning behaviour of red colorants in CGA was measured in terms of recovery in the aphron phase ( $\eta$, Eq. (4)) and partition coefficient (K, Eq. (5)):

$\eta=\frac{A b s_{490 I N I} \times\left(V_{A P}+V_{F B}\right)-\left(A b s_{490 L P} \times V_{L P}\right)}{A b s_{490 I N I} \times\left(V_{A P}+V_{F B}\right)} \times 100$
$K=\frac{A b s_{490 A P}}{A b s_{490 L P}}=\frac{\frac{A b s_{490 I N I} \times\left(V_{A P}+V_{F B}\right)-\left(A b s_{490 L P} \times V_{L P}\right)}{V_{A P}}}{A b s_{490 L P}}$

where $\mathrm{Abs}_{490 I N I}, \mathrm{Abs}_{490 \mathrm{AP}}$ and $\mathrm{Abs}_{490 \mathrm{LP}}$ are the red colorants absorbance at $490 \mathrm{~nm}$ initial, in the aphron and in the liquid phase, respectively. $V_{\mathrm{AP}}, V_{\mathrm{LP}}$ and $V_{\mathrm{FB}}$ are the volume of liquid in the aphron phase, liquid phase and fermented broth, respectively.

Selectivity of separation for red colorants in relation to proteins $\left(S e_{P}\right.$, Eq. (6)) and sugars (Se, Eq. (7)) was defined as:

$S e_{P}=\frac{K}{K_{P}}=\frac{K}{\frac{P_{A P}}{P_{L P}}}$
$S e_{S}=\frac{K}{K_{S}}=\frac{K}{\frac{S_{A P}}{S_{L P}}}$

where $K$ is partition coefficient of red colorants, $K_{P}$ and $K_{S}$ is the partition coefficient of proteins and sugar, respectively. $P_{\mathrm{AP}}$ and $P_{\mathrm{LP}}$ are the concentration of proteins in the aphron and liquid phase, respectively. $S_{\mathrm{AP}}$ and $S_{\mathrm{LP}}$ are the concentration of sugar in the aphron and liquid phase, respectively.

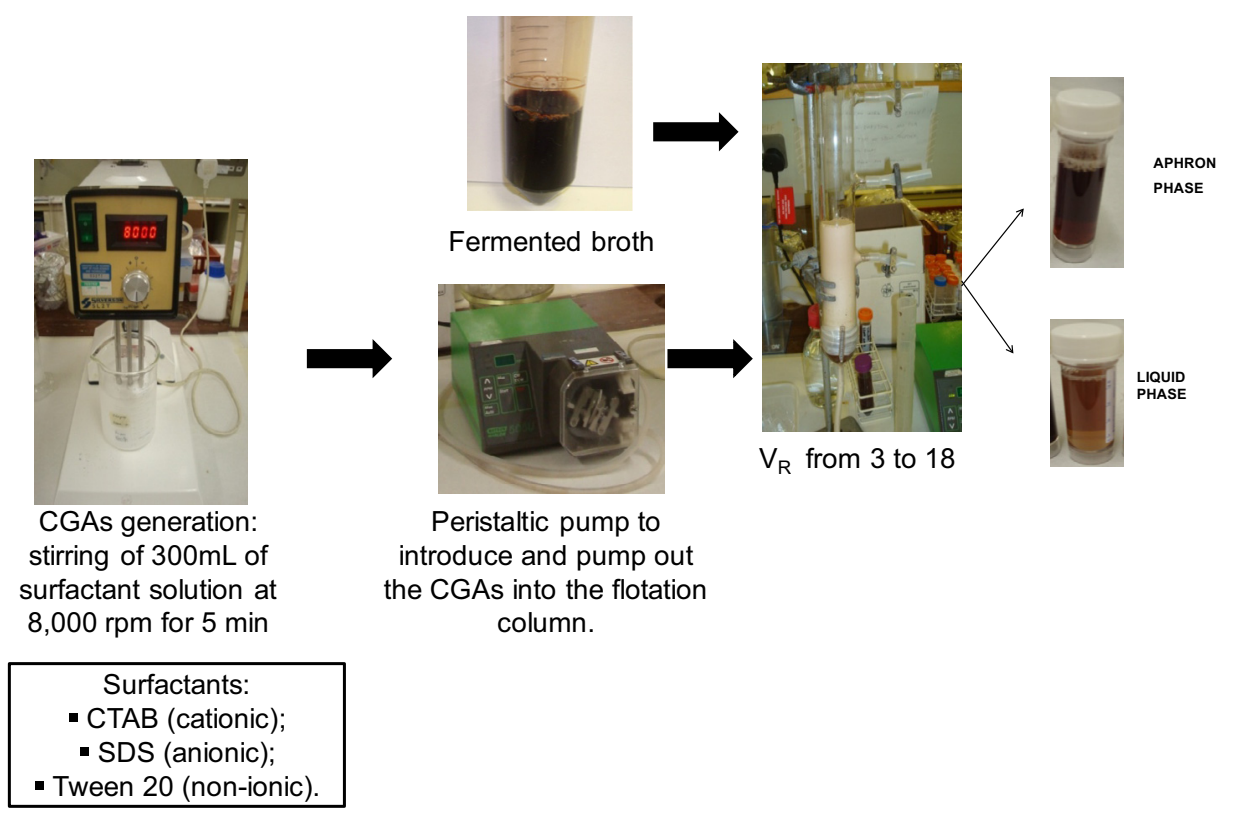

Fig. 1. The scheme of the experiment. 


\subsection{Red colorants interactions with surfactants}

The interaction of natural colorants with the surfactants, Tween $20(20 \mathrm{mM})$, SDS (2 mM) and CTAB (2 mM) at pH 6.9, 8.0, 9.0 and 10.0 was evaluated. The experiments were carried out as follows: for each surfactant, fermented broth of $T$. amestolkiae was added reaching a final absorbance of $2.00 \mathrm{UA}_{490 \mathrm{~nm}}$ to red colorants. Solutions were homogenized in a cuvette and inserted in a thermoregulated cuvette holder at $25{ }^{\circ} \mathrm{C}$. Absorbance was determined at 0,1 , 3,6 and $24 \mathrm{~h}$.

\subsection{Relative solubility of red colorants in organic solvents}

Red colorants solubility in organic solvents was carried out according the methodology described by Craft and Soares [34] with some modifications. The solvents studied were ethanol absolute, methanol, ethyl acetate, propanol and hexane. The experiments were carried out as following: $0.5 \mathrm{~mL}$ of fermented broth with $2.00 \mathrm{UA}_{490 \mathrm{~nm}}$ was added to $4 \mathrm{~mL}$ of each solvent listed above. The samples were agitated for $15 \mathrm{~min}$ and after each solution was filtered through a $0.2-\mu \mathrm{m}$ membrane. The background absorbance of each solution was subtracted using the appropriate solvent.

\subsection{Partition coefficient 1-octanol/water}

The experiments to calculate the octanol-water partition coefficient $\left(P_{\mathrm{OW}}\right)$ of red colorants from fermented broth was carried out using the methodology of Shake Flask Method [35]. Before the partition coefficient determination, 1-octanol and water were saturated at $25{ }^{\circ} \mathrm{C}$. To do this, two large stock bottles containing 1octanol and a sufficient quantity of water, and the other containing water and a sufficient quantity of 1 - were stirred for $24 \mathrm{~h}$ on an orbital shaker at $125 \mathrm{rpm}$. Thus, $2 \mathrm{~mL}$ of fermented broth $(2.00$ $\left.\mathrm{UA}_{490 \mathrm{~nm}}\right)$ at $\mathrm{pH}$ range of 4.0-10.0 was added to three different volume ratio of n-octanol to water $(0.5,1.0$ and 2.0). The separation of the two phases was achieved by centrifugation $(5,000 \mathrm{rpm}$ during 15 min at $25^{\circ} \mathrm{C}$ ). $P_{\text {ow }}$ was defined as the ratio of the equilibrium concentration of the substance in the octanol-rich phase to that in the water-rich phase (Eq. (8)). The red colorants absorbance was measured in both phases and the results were expressed as logarithm of $P_{\mathrm{OW}}, \log P_{\mathrm{OW}}$.

$P_{O W}=\frac{A b s_{4900 P}}{A b s_{490 A P}}$

where $\mathrm{Abs}_{4900 \mathrm{P}}$ and $\mathrm{Abs}_{490 \mathrm{AP}}$ are the red colorants absorbance at $490 \mathrm{~nm}$ in the octanol and aqueous phase, respectively.

\subsection{Analytical methods}

\subsubsection{Red colorants determination}

Since the concentration of colorants is usually represented by color value based on the absorbance spectrum [36], the red colorants in each phase were estimated by spectrophotometric analysis. To determine the wavelength at maximum absorption of red colorants the fermented broth was scanned in the wavelength range $=220-600 \mathrm{~nm}$ at room temperature. Thus, the absorbance of the red colorants was fixed at $490 \mathrm{~nm}$, which is in accordance with the literature $[37,38]$.

\subsubsection{Total protein determination}

Total protein concentration was determined using the bicinchoninic acid method (BCA), compatible with surfactants.

\subsubsection{Total sugar determination}

The total sugar concentration presented in the fermented broth was measured using refractometer instrument. The instrument was calibrated with sucrose and D-glucose solutions. To the samples, $800 \mu$ of fermented broth had its refractive index measured by a tube type polarimeter (Optical Activity ltd) and from the standard curve calculate the sample total sugar concentration was measured. So, it was possible to measure the total sugar in the liquid and aphron phase.

\subsection{Statistic}

All experiments were performed in triplicate, and the standard deviations and confidence intervals calculated. The limit of significance for the statistical analysis was set at $95 \%$.

\section{Results and discussion}

\subsection{CGA characterization}

The main goal of this study was to recover red colorants from the fermented broth of T. amestolkiae using CGA generated by using different surfactant solutions. Initial experiments were conducted to characterize CGA in terms of gas hold-up $(\varepsilon)$ and stability. CGA were generated with the anionic surfactant SDS, nonionic surfactant Tween 20, and cationic surfactant CTAB at four different $\mathrm{pH}$ values $(6.9,8.0,9.0$, and 10.0) and their characteristics were measured.

The concentration used for ionic surfactants was chosen based on work carried out by other researchers in the group [28]. According to this work CTAB concentrations higher than $2 \mathrm{mM}$ did not cause a significant change in the colloidal stability. The same concentration was chosen for the anionic surfactant SDS in order to make comparisons. The concentration of $20 \mathrm{mM}$ for Tween 20 was chosen based on the results reported by Dermiki et al. [14], who found that at $2 \mathrm{mM}$, the half-life was low (166 s) and at $20 \mathrm{mM}$ half-life was $582 \mathrm{~s}$ and gas hold-up $=63 \%$ which is in agreement with results obtained here (Table 1 ). So, higher concentration of nonionic surfactants are necessary to produce stable CGA when compared to ionic surfactants. This can be attributed to the larger surface area per molecule and the absence of highly charged surface films in the films produced by nonionic surfactants $[39,14]$. The half-life obtained for Tween 20 in the present work was higher than that reported by Dermiki et al. [14] with Tween 60. This may be attributed to the different chemical structures of the surfactants, because Tween 20 is sorbitan monolaurate, while Tween 60 is sorbitan monostearate.

As can be seen in Table 1, the surfactant type had no significant effect on gas hold-up, which remained at around $65 \%$. The results obtained with $\mathrm{CTAB}$ at different $\mathrm{pH}$ values are consistent with those reported by Fuda et al. [28], who performed experiments at $\mathrm{pH} 4.0,6.0$, and 8.0, and found no appreciable changes in CGA properties with varying $\mathrm{pH}$.

With regard to the half-life, CGA generated with SDS showed the lowest stability probably because of its anionic characteristics.

Table 1

Effect of SDS (2 mM), Tween $20(20 \mathrm{mM})$ and CTAB $(2 \mathrm{mM})$ in different pHs on the CGA characterization.

\begin{tabular}{lllr}
\hline Surfactant & & Air Hold-up, $\varepsilon(\%)$ & Half-life, $\tau(\mathrm{s})$ \\
\hline SDS $(2 \mathrm{mM})^{\mathrm{a}}$ & & $65.96 \pm 1.66$ & $348.80 \pm 29.93$ \\
Tween $20(20 \mathrm{mM})^{\mathrm{a}}$ & & $64.66 \pm 0.74$ & $553.40 \pm 11.83$ \\
CTAB $(2 \mathrm{mM})$ & pH 6.90 & $64.71 \pm 1.07$ & $402.38 \pm 33.53$ \\
& pH 8.0 & $64.68 \pm 0.37$ & $381.25 \pm 5.46$ \\
& pH 9.0 & $63.53 \pm 1.75$ & $407.20 \pm 19.95$ \\
& pH 10.0 & $66.27 \pm 0.51$ & $454.00 \pm 23.86$
\end{tabular}

a The SDS and Tween 20 solutions were prepared in deionised water with final $\mathrm{pH}$ of 6.9 . 
$\mathrm{CTAB}$, independently, of $\mathrm{pH}$, had a lower stability than that of Tween 20. Therefore, Tween 20 had the highest stability, which was $59 \%$ and $45 \%$ higher than that of SDS and CTAB at $\mathrm{pH} 8.0$, respectively. It is expected that ionic surfactants increase the colloidal stability because the charge of the surfactants promotes repulsive and stabilizing forces between aphrons, delaying their coalescence and resulting in a higher-stability suspension [28]. However, as we worked with a Tween 20 concentration 10 times higher than that of the ionic surfactants, we cannot make comparisons in this sense.

Generally, in order to produce stable CGA, the surfactant concentration has to exceed the critical micellar concentrations (CMC) of the surfactant, because under these conditions, a viscous liquid-crystalline phase in the bulk solution is formed and the drainage of the foam is delayed [39,14]. The CMC of Tween 20, CTAB and SDS in water is $0.08,0.90$ and $8.08 \mathrm{mM}$, respectively [27]. It is interesting that although we worked with SDS concentration lower than its $C M C$ the results of air hold-up was close to the ones achieved with CTAB and Tween 20.

The concentration of $2 \mathrm{mM}$ for CTAB was found to be optimum in our previous studies (data no shown). However, studies comparing the CGA characteristics at different pHs have not yet been conducted. The results showed that CGA at pH 10 were most stable. So, under basic conditions higher-stability foams are generated by CTAB.

To conclude, since similar values of air hold-up and half-life were achieved with SDS and CTAB at $2 \mathrm{mM}$ and based on our previous studies this concentration was chosen for the partitioning studies with ionic surfactants while $20 \mathrm{mM}$ was chosen for Tween 20.

\subsection{Red colorants interaction to surfactants}

Surfactants are widely used for many purposes in almost every sector of modern industry. Furthermore, some of them are used in many formulations of petrochemical, chemical, pharmaceutical, food, cosmetic and textile industries [40]. An important property of micelles that is highly significant in the pharmaceutical and food industries is their ability to increase the solubility of sparingly soluble substances in water [18]. In this way, by using a surfactant for the separation step (CGA's) which enhances the solubility and the stability of the red colorants, it is possible to design an integrative process where the recovery and stabilization (formulation) of the colorants is achieved in one step. Fig. 2 shows the results of colorants interaction with Tween 20, SDS and CTAB at pH 6.9, 8.0, 9.0 , and 10.0 after $1,3,6$, and $24 \mathrm{~h}$.

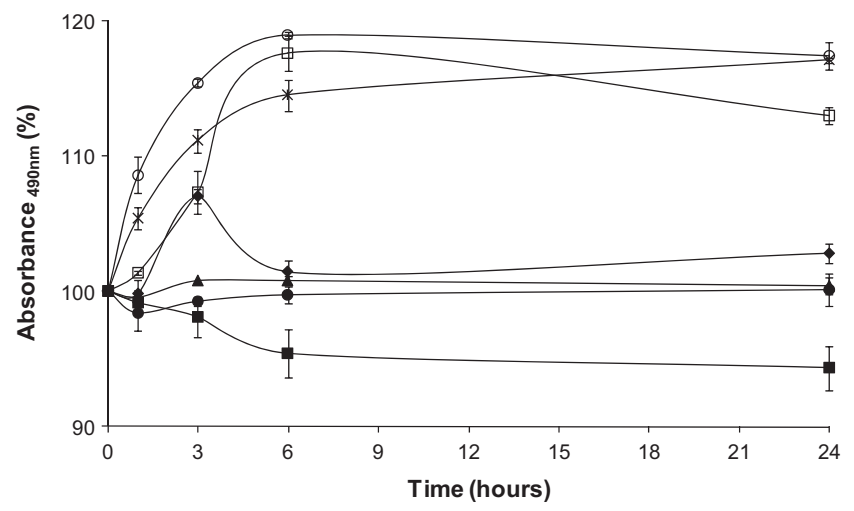

Fig. 2. Interaction of red colorants from fermented broth of $T$. amestolkiae at $25{ }^{\circ} \mathrm{C}$ with surfactants and pHs during $24 \mathrm{~h}$ : standard (without surfactant, $\bullet$ ), $20 \mathrm{mM}$

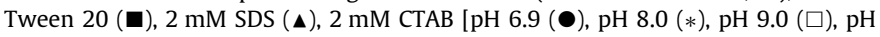
$10.0(\bigcirc)$ ]. The error bars represent $95 \%$ confidence limit for the measurements.
Red colorants were stable in the presence of the surfactant since no precipitation was observed. Interestingly it was observed that the absorbance values increased (by about $15 \%$ ) with CTAB at $\mathrm{pH}$ $8.0,9.0$, and 10.0 over time as compared to the standard solution whereas the absorbance decreased in the presence of Tween 20 and did not significantly change in the presence of SDS. This was attributed to some sort of association or complexation between the red colorants and surfactant monomers which happens mainly by electrostatic and hydrophobic interactions [41-44]. RangelYagui et al. [18] reported the important effect of hydrophobicity on the micellar binding of carminic acid with various surfactants. According to Gokturk and Tunçay [45] the interaction between colorant and surfactant is dependent on the chemical structure of the colorant and surfactant and it can result in two types of interactions: formation of a complex between surfactant and colorant or incorporation of the colorant to the surfactant micelle when the surfactant concentration is higher than its critical micellar concentration (CMC). As reported by Rangel-Yagui et al. [18], the pH of micellar solutions can also show a significant influence on the extent of solubilization and stabilization of the biomolecules, since it can change the equilibrium between the ionized and molecular forms of some compounds. Ionic surfactants can interact not only by electrostatic interactions but also through hydrophobic interactions [44]. This is clearly demonstrated in the results obtained with CGA generated from different surfactants (see Section 3.4).

\subsection{Solubility and octanol/water partition of red colorant from the fermented broth of $P$. purpurogenum in different solvents}

It is expected that the red colorants produced by T. amestolkiae presents similar chemical structure with the Monascus colorants and be water soluble as well. The relative solubility of red colorants studies in ethanol, methanol, ethyl acetate, propanol, and hexane was determined. The red colorants were most soluble in polar organic solvents. The polarity of organic solvents increases in following sequence: hexane < ethyl acetate < propanol < ethanol <methanol. Among the alcohols studied, the lower solubility of red colorants was achieved in propanol. It can be attributed to the longer carbon chain of propanol, resulting in a lower polarity. Red colorants were found to be completely insoluble in hexane and partially miscible in ethyl acetate. These results shows the hydrophilic nature of the red colorants. This is further confirmed by the partitioning of the colorants in the octanol/water system ( $\log P_{\text {ow }}$ values) obtained at varying $\mathrm{pH}$ conditions (Fig. 3 ).

The octanol/water partition coefficient $\left(\log P_{\mathrm{OW}}\right)$ of red colorants was negative in the whole $\mathrm{pH}$ range tested, ie: these molecules partition preferentially into the aqueous phase. Moreover log $P_{\text {ow }}$ decreased with increase in $\mathrm{pH}$. At basic $\mathrm{pH}$ hydroxyl groups in the colorants will be ionized which will make the molecules more hydrophilic and hence will partition preferentially into the water

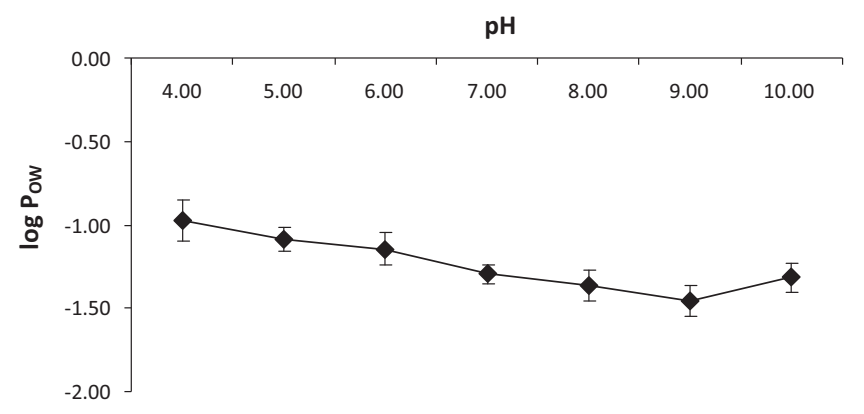

Fig. 3. $\log \mathrm{P}_{\mathrm{OW}}$ of red colorant presented in the fermented broth of T. amestolkiae at $\mathrm{pH}$ range of $4.0-10.0$. The error bars correspond to $95 \%$ confidence level. 
phase. This is important information to take into account in the development of an strategy for the extraction of red colorants from T. amestolkiae.

\subsection{Partitioning of colorants from the fermented medium}

Depending on the chemical characteristics of bioproducts, the surfactant's charge can have a strong influence on the recovery. In this respect, electrostatic interactions between charged bioproducts and charged surfactants can be exploited to enhance the yield and selectivity using CGA, as one of the major problems with colorant production is their purification [46]. In previous studies [14], the effect of volumetric ratio $\left(V_{R}\right)$ was shown to be also an important operating parameter in the recovery of other bioproducts. In the present study, $V_{R}$ was varied between 3 and 18 and the CGA was generated by the surfactants SDS, CTAB, and Tween 20.

According to results shown in Fig. 4A, an increase in $V_{R}$ had a strong influence on the recovery, mainly with surfactants CTAB and Tween 20 . The lowest recovery was obtained at a $V_{R}$ of 3, presumably because at this ratio, there was not enough CGA to carry all the colorant molecules and to provide enough surface area for their adsorption. This is further supported by Fig. 4B where is shown that increasing the $V_{R}$ from 3 to 12 results in increased recovery and partition coefficient. This may be because at higher $V_{R}$ more CGA will be available for the recovery. However at very high $V_{R}$ a decrease in recovery was observed for Tween 20 and a decrease in partitioning coefficient (to about 1 ) for all surfactants. At high $V_{R}$ a larger volume of CGA which means a larger volume of surfactant solution (within the CGA phase) is available for the
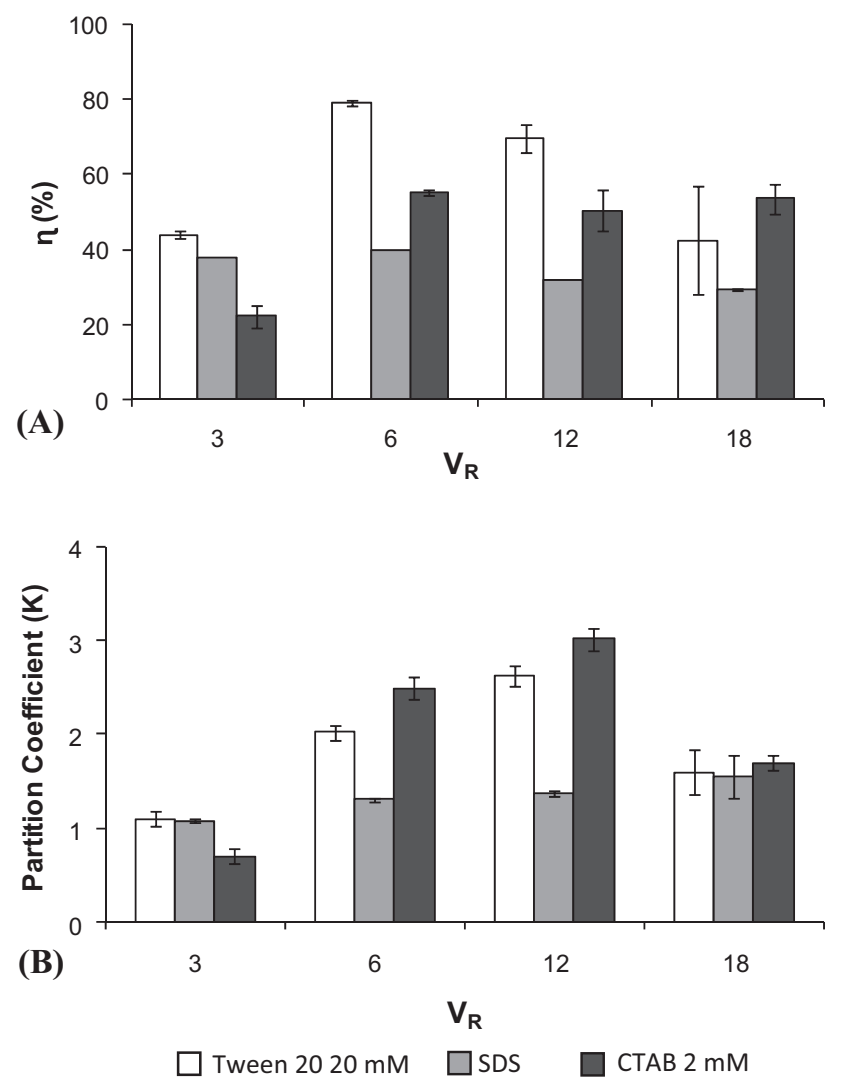

Fig. 4. Comparison of recovery $(\eta)(A)$ and partition coefficient $(K)$ (B) of red colorants from fermented broth using CGA produced by $20 \mathrm{mM}$ Tween 20), $2 \mathrm{mM}$ SDS and 2 mM CTAB as a function of the $V_{R}$. The error bars represent $95 \%$ confidence limit for the measurements. solubilisation of the colorants in the surfactant micellar solution. In this way, there is a competition between solubilisation and flotation by the CGA. This could explain the decrease in the partitioning observed for all surfactants at the highest $V_{R}$.

The highest recovery (78.83\%) was obtained with Tween 20 at $V_{R}$ 6. The largest partitioning (3.00) was obtained with CTAB at $V_{R} 6$ followed by Tween 20 at the same $V_{R}(2.70)$. Clearly the recovery with SDS was the lowest which could be explained based on repulsive electrostatic interactions between the colorants and the anionic surfactant. This is consistent with our hypothesis that the red colorants are negatively charged and that both electrostatic and hydrophobic interactions play an important role in the recovery of the red colorants with CGA. Similarly in a previous study by our group on another natural colorant, norbixin (negatively charged), we found that maximum recovery was obtained with the $C T A B$ and very poor recoveries with the anionic surfactant AOT [22].

As Tween 20 and CTAB showed the best results further experiments were carried out with CGA generated with these surfactants and at optimum $V_{R}$ conditions, 6 and 12 .

\subsection{Partitioning of colorants from the fermented medium using CGA generated by CTAB at different $\mathrm{pHs}$}

Recovery experiments were carried out at different $\mathrm{pH}$ values $(6.9,8.0,9.0$, and 10.0). More basic $\mathrm{pH}$ values were chosen because stability studies for red colorants at $\mathrm{pH} 3.0-10.0$ showed that these colorants are more stable under basic conditions [11]. The results of recovery and partition coefficient are shown in Fig. 5A and B, respectively.

Recovery increased with $\mathrm{pH}$ and similar recovery (about 70\%) was obtained in the alkali range ( $\mathrm{pH} \mathrm{8.0-10.0)} \mathrm{(Fig.} \mathrm{5A).} \mathrm{The} \mathrm{parti-}$ tion coefficient followed a similar trend with a maximum at $\mathrm{pH} 9.0$ and at $V_{R} 12$. These results confirmed that the stronger electrostatic attractive interactions between the CTAB and colorants led to their increased recovery and partitioning into the CGA phase. Besides, as found in the partitioning experiments (Fig. 3) log Pow had its
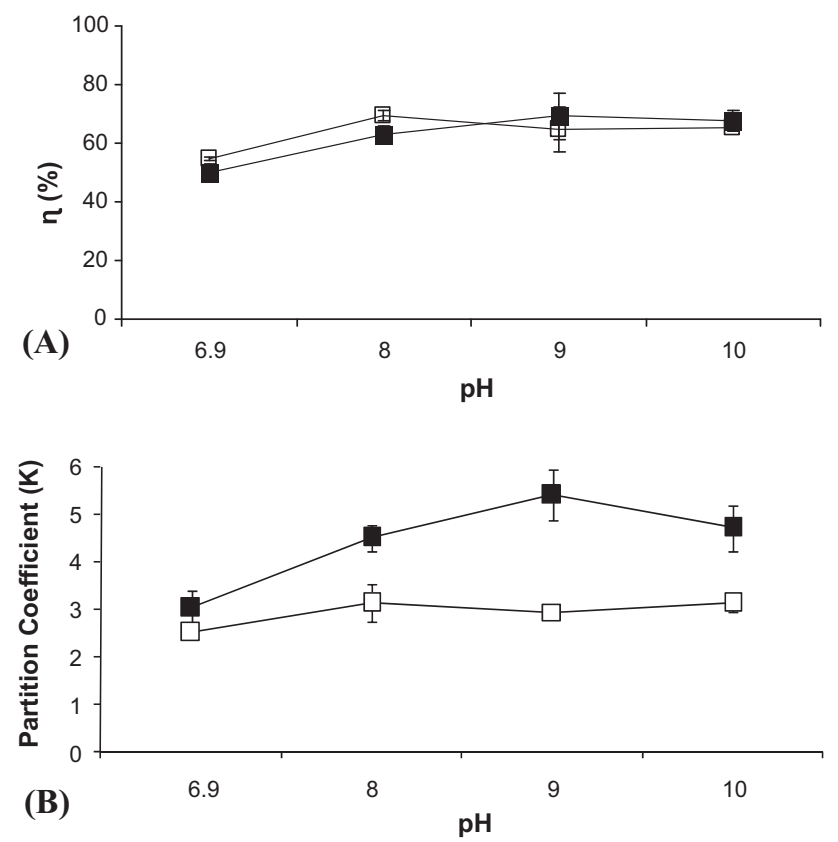

Fig. 5. Study of the effect of $\mathrm{pH}$ on the recovery $(\eta)(A)$ and partition coefficient $(K)$ (B) of red colorant from fermented broth using CGA generated by CTAB $2 \mathrm{mM}$ in the $V_{R}$ at $6(\square)$ and $12(\square)$ at different pHs (6.9, 8.0, 9.0 and 10.0). The error bars represent $95 \%$ confidence limit for the measurements. 
lowest value at $\mathrm{pH} 9.0$, at the $\mathrm{pH}$ that the highest partitioning was obtained. These results are also in agreement with those reported by Santos-Ebinuma et al. [38], who studied the partitioning of red colorants in aqueous two-phase system formed by PEG and sodium polyacrylate (NaPA), a strongly negatively charged polyelectrolyte. All the above results show that the extraction of red colorants into CGA generated by CTAB, a cationic surfactant, was partly driven by electrostatic interactions.

Moreover higher partition coefficients were achieved at $V_{R} 12$ than at 6 (Fig 5B). Higher volumetric ratios correspond to higher mass ratios of surfactants to solids in the feed, resulting in more surfactant molecules available for the partition.

As can be seen at Table 2, selectivity measured in relation to proteins $\left(S e_{\mathrm{P}}\right)$ was more influenced by $V_{R}$ than by $\mathrm{pH}$. The $V_{R}$ of 12 provided $S e_{P}$ of around 3.0, which was $50 \%$ higher than the one obtained at $V_{R} 6$. The $\mathrm{pH}$ of 9.0 afforded the lowest values of $S e_{\mathrm{p}}$ under all conditions evaluated. It is interesting that not only the red colorants but also the proteins were more attracted to the aphron phase. However, this result can affect other steps of red colorant recovery from the fermented broth because CGA affords a partial separation of proteins from the fermented broth.

Selectivity measured in relation to sugar $\left(\mathrm{Se}_{S}\right)$ (Table 2) was strongly affected by both $\mathrm{pH}$ and $V_{R}$. At $V_{R} 12$ and $\mathrm{pH} 9.0$, a $S e_{\mathrm{S}}$ of 7.2 was obtained, and the recovery values obtained under these conditions were $138 \%, 33 \%$, and $53 \%$ higher than those obtained at $\mathrm{pH} 6.9,8.0$, and 10.0, respectively. This is because of the stronger attraction of red colorants to CTAB than the sugars present in the fermented broth.

With higher selectivity, better separation of target biomolecules is observed, and CGA generated by CTAB produced good results mainly at $\mathrm{pH}$ 9.0. Further investigations such as variations in drainage time and different CTAB concentrations can be carried out in order to optimize the results above. Nevertheless, the results presented with CTAB already demonstrate the potential of this purification strategy.

\subsection{Partitioning of colorants from the fermented medium using CGA generated by Tween 20 (20 mM)}

As the recovery of red colorants using Tween 20 seems to be driven by hydrophobic interactions, the strategy to dissolve the colorants in Tween 20 and ethanol prior to CGA application was evaluated against the 'standard' ie, CGA applied to aqueous red colorants solution. Both results are presented in Fig. 6 .

Partial dissolution of red colorants in a solution of Tween 20 (20 $\mathrm{mM}$ ) was proposed before the contact with CGA generated by Tween 20, because, hypothetically, the red colorants could be covered by the surfactant, resulting in their increased recovery. The results showed that the experiments proposed did not improve recovery or the partition coefficient. In Fig. $6 \mathrm{~B}$, it can be seen that $V_{R}$ of 12 promotes higher values of $K$ at the 20:80 proportion and red colorants without dilution. This can be explained if we consider that the recovery is a parameter that considers the volume in both phases and, as can be seen in Table 3 , the ratio $V_{\mathrm{LIO}} / V_{\mathrm{APHRON}}$ under
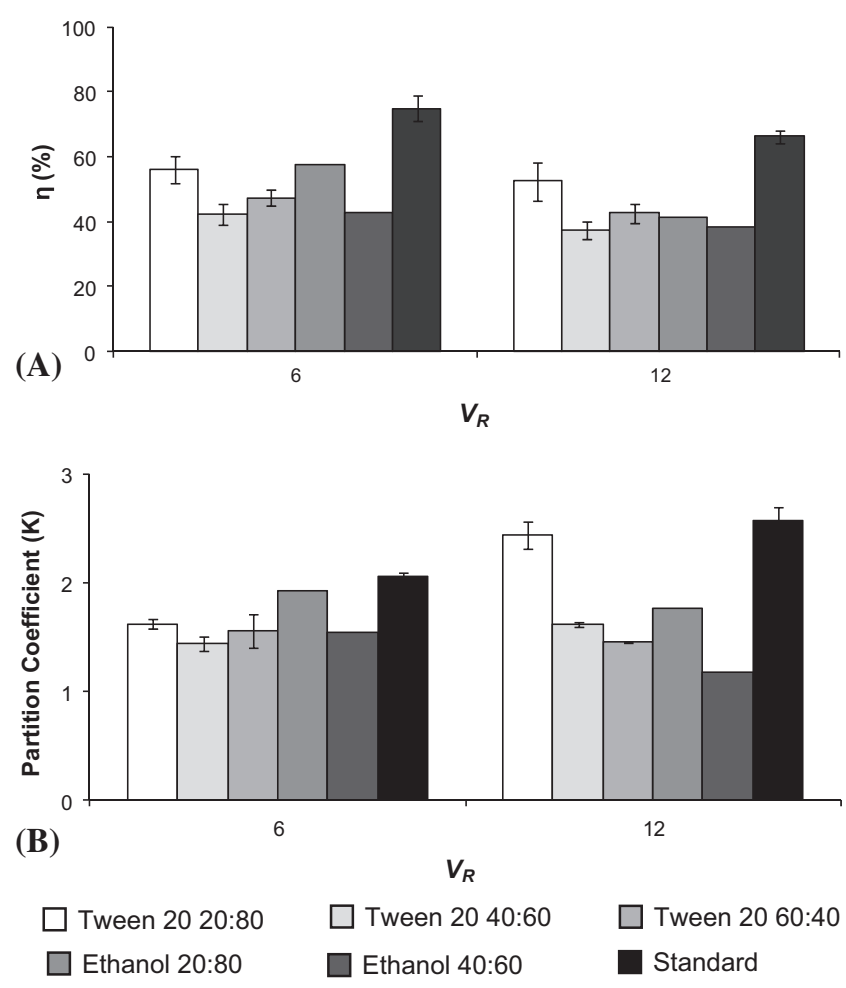

Fig. 6. Recovery $(\eta)(A)$ and partition coefficient $(K)$ (B) of red colorant from fermented broth dispersed in Tween $20(20: 80,40: 60$ and 60:40 v/v), ethanol (20:80 and 40:60 v/v) and standard (without dilution) using CGA generated by $20 \mathrm{mM}$ Tween 20 as a function of the $V_{R}$. The error bars represent $95 \%$ confidence limit for the measurements.

standard conditions is twofold higher at $V_{R} 12$ than that at $V_{R} 6$. At any rate, the partial dissolution of red colorants in a solution of Tween 20 did not promote the expected results. The best values of recovery (78\%) and partition coefficient (2.6) were achieved under standard experimental conditions.

The dissolution of red colorants in ethanol did not promote an increase in the extraction parameters. An increase in the percent volume of ethanol in the solution led to a decrease in all the parameters analyzed. Save and Pangarkar [47] reported that the addition of alcohol resulted in the localized reduction of the surface tension to exceptionally low values to the extent of its subsequent rupture, causing the destabilization of CGA. Therefore, when the solvent/fermented broth mixture ratio is $40: 60$, the presence of ethanol will promote the destabilization of CGA. The partition coefficient in the standard at $V_{R} 12$ was $46 \%$ higher than that of the red colorants dispersed in ethanol (20:80).

The addition of either Tween 20 or ethanol produced a $S e_{\mathrm{P}}$ (Table 3) higher than that obtained under standard conditions at 20:80 and $V_{R}$ 12. However, an increase in the concentration of these solvents decreased this parameter. $S e_{\mathrm{P}}$ did not have much of an influence on the results. $S e_{S}$ was higher under the standard

Table 2

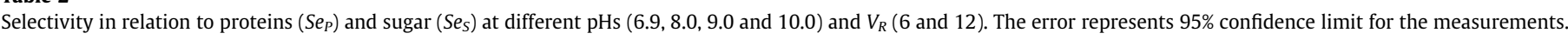

\begin{tabular}{|c|c|c|c|c|c|c|}
\hline \multirow[t]{2}{*}{$\mathrm{pH}$} & \multicolumn{2}{|c|}{$\begin{array}{l}V_{\mathrm{LIQ}} / V_{\text {APHRON }} \\
V_{R} \\
\end{array}$} & \multicolumn{2}{|l|}{$\begin{array}{l}S e_{P} \\
V_{R} \\
\end{array}$} & \multicolumn{2}{|l|}{$\begin{array}{l}\mathrm{Se}_{S} \\
\mathrm{~V}_{R} \\
\end{array}$} \\
\hline & 6 & 12 & 6 & 12 & 6 & 12 \\
\hline 6.9 & 2.1 & 3.0 & $1.94 \pm 0.16$ & $2.18 \pm 0.25$ & $2.04 \pm 0.01$ & $3.02 \pm 0.36$ \\
\hline 8 & 1.4 & 2.8 & $2.32 \pm 0.18$ & $3.18 \pm 0.36$ & $3.12 \pm 0.40$ & $5.39 \pm 0.61$ \\
\hline 9 & 1.6 & 2.4 & $2.02 \pm 0.18$ & $3.75 \pm 0.49$ & $3.67 \pm 0.31$ & $7.20 \pm 0.48$ \\
\hline 10 & 1.5 & 2.2 & $2.17 \pm 0.16$ & $3.04 \pm 0.49$ & $3.13 \pm 0.19$ & $4.71 \pm 0.48$ \\
\hline
\end{tabular}


Table 3

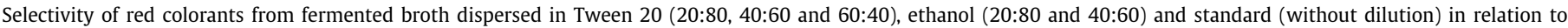
proteins $\left(S e_{P}\right)$ and sugar $\left(S e_{S}\right)$ at different $V_{R}(6$ and 12$)$. The error represents $95 \%$ confidence limit for the measurements.

\begin{tabular}{|c|c|c|c|c|c|c|c|}
\hline \multirow[t]{2}{*}{ Condition } & & \multicolumn{2}{|c|}{$\begin{array}{l}V_{\mathrm{LIQ}} / V_{\mathrm{APHRON}} \\
V_{R}\end{array}$} & \multicolumn{2}{|l|}{$\begin{array}{l}S e_{P} \\
V_{R}\end{array}$} & \multicolumn{2}{|l|}{$\begin{array}{l}\mathrm{Se}_{S} \\
V_{R}\end{array}$} \\
\hline & & 6 & 12 & 6 & 12 & 6 & 12 \\
\hline Standard & & 1.5 & 3.2 & $1.35 \pm 0.02$ & $1.34 \pm 0.17$ & $2.01 \pm 0.06$ & $2.70 \pm 0.35$ \\
\hline \multirow[t]{3}{*}{ Tween 20 (20 mM) } & $20: 80$ & 1.3 & 2.3 & $1.22 \pm 0.07$ & $1.73 \pm 0.17$ & $1.62 \pm 0.08$ & $2.60 \pm 0.24$ \\
\hline & $40: 60$ & 2.0 & 2.7 & $1.12 \pm 0.12$ & $1.16 \pm 0.07$ & $1.44 \pm 0.13$ & $1.70 \pm 0.05$ \\
\hline & $60: 40$ & 1.7 & 2.0 & $1.22 \pm 0.25$ & $1.11 \pm 0.03$ & $1.86 \pm 0.11$ & $1.77 \pm 0.16$ \\
\hline \multirow[t]{2}{*}{ Ethanol } & $20: 80$ & 2.0 & 2.7 & $1.50 \pm 0.11$ & $1.52 \pm 0.04$ & $1.74 \pm 0.18$ & $1.70 \pm 0.09$ \\
\hline & $40: 60$ & 1.7 & 2.0 & $1.29 \pm 0.09$ & $1.22 \pm 0.08$ & $1.50 \pm 0.03$ & $1.23 \pm 0.08$ \\
\hline
\end{tabular}

conditions than that observed with the addition of either Tween 20 or ethanol. Dissolving the fermented broth in ethanol resulted in the lowest $\mathrm{Se}_{\mathrm{S}}$ values.

In summary best results were obtained with CGA generated by Tween 20 and CTAB and when applied directly to the aqueous colorant solution (without prior dissolution in ethanol or Tween 20). It is interesting that at $V_{R} 12$, the highest values for some of the extraction parameters were obtained not only with Tween 20 but also with $\mathrm{CTAB}$. In $\mathrm{CTAB}$, the extraction of red colorants is driven mainly by electrostatic interactions between red colorants molecules and CTAB monomers. In Tween 20, the separation is mainly driven by hydrophobic interactions. If the surfactant concentration is not high enough to develop the association between the hydrophilic surfactant moiety and red colorants molecules, the latter will stay in the liquid phase, which has the more hydrophilic environment, thus reducing the extraction.

Overall CTAB led to better results than Tween 20. However, depending on the application of the red colorants, the use of Tween 20 is more interesting than CTAB because of the lower toxicity of the former. CTAB has a $\mathrm{LD}_{50}=410 \mathrm{mg} / \mathrm{kg}$, which is too high for use in the food industry, for example, but can be used to dye clothes. Nonionic surfactants, on the other hand, can be advantageous to use as they are nontoxic and are used in the formulation of medicines; hence, it may not be essential to remove them from the CGA phase and, at the same time, they could help to formulate the final product [27].

\section{Conclusions}

The partitioning behavior of red colorants from the fermented broth of T. amestolkiae in colloidal gas aphrons (CGA) was investigated. Solubility studies showed that the red colorants have more hydrophilic characteristics. With regard to recovery experiments, CTAB and Tween 20 led to better results than SDS. Among these surfactants, when the recovery tests were performed with CTAB within $\mathrm{pH}$ range 6.9-10.0, the colorants were strongly attracted to the positively charged molecules. So, electrostatic interactions between the negatively charged red colorants and the cationic surfactant led to the highest recoveries. Tween 20 also led to high recoveries but driven mainly by hydrophobic interactions. In the case of CGA generated by Tween 20 prior solubilisation of the colorants in ethanol or Tween 20 solutions did not enhance their recovery. Interestingly the highest selectivity (both in terms of sugar and protein) was obtained with $\mathrm{CTAB}$ at $\mathrm{pH}=9$ and $\mathrm{Vr}=12$, conditions at which maximum recovery of the colorants was obtained. Since high recoveries were achieved with both CTAB and Tween 20 surfactants, the choice of the surfactant will be based on the final application of the red colorants. Finally, this work demonstrated the potential of CGA as an alternative to liquid extraction methods which can be applied at the recovery stage in the separation of colorants from a fermentation culture.

\section{Acknowledgements}

The authors are gratefully to Coordination for the Improvement of Higher Education Personnel - CAPES by the financial support in the form of scholarship to V.C. Santos-Ebinuma (Process BEX 0321/11-7), São Paulo Research Foundation - FAPESP (Process 08/58280-0) and the Department of Food and Nutritional Sciences of University of Reading.

\section{References}

[1] S.A.S. Mapari, K.F. Nielsen, T.O. Larsen, J.C. Frisvad, A.S. Meyer, U. Thrane, Exploring fungal biodiversity for the production of water-soluble pigment as potential natural food colorants, Curr. Opin. Biotech. 16 (2005) 231-238.

[2] W. Hailey, R. Zhifang, L. Ping, G. Yanchang, L. Guosheng, Y. Jianming, Improvement of the production of a red pigment in Penicillium sp. HSD07B synthesized during co-culture with Candida tropicalis, Bioresource Technol. 102 (2011) 6082-6087.

[3] S. Kongruang, Grown Kinetics of biopigment production by Thai isolated Monascus purpureus in a stirred tank bioreactor, J. Ind. Microbiol. Biotechnol. 38 (2011) 93-99.

[4] P. Velmurugan, S. Kamala-Kannan, V. Balachandar, P. Lakshmanaperumalsamy, J.-C. Chae, B.-T. Oh, Natural pigment extraction from five filamentous fungi for industrial applications and dyeing leather, Carbohyd. Polym. 79 (2010) 262268.

[5] V.C. Santos-Ebinuma, M.F.S. Teixeira, A. Pessoa Jr, Submerged culture conditions for the production of alternative natural colorants by a newly isolated Penicillium purpurogenum DPUA 1275, J. Microbiol. Biotechn. 23 (2013) 802-810.

[6] S.A.S. Mapari, U. Thrane, A.S. Meyer, Fungal polyketide azaphilone pigments as future natural food colorants?. Trends Biotechnol 28 (2010) 300-307.

[7] S.A.S. Mapari, A.S. Meyer, U. Thrane, Colorimetric Characterization for Comparative analyses of Fungal Pigments and Natural Food Colorants, J. Agric. Food Chem. 54 (2006) 7027-7035.

[8] S.A.S. Mapari, A.S. Meyer, U. Thrane, J.C. Frisvad, Identification of potentially safe promising fungal cell factories for the production of polyketide natural food colorants using chemotaxonomic rationale, Microb Cell Fact. 24 (2009) 1 15.

[9] J.R. Celestino, L.E. Carvalho, M.P. Lima, A.M. Lima, M.M. Ogusku, J.V.B. Souza, Bioprospecting of Amazon soil fungi with the potential for pigment production, Process Bioch. 49 (2014) 569-575.

[10] M.F.S. Teixeira, M.S. Martins, J. Da Silva, L.S. Kirsch, O.C.C. Fernandes, A.L.B. Carneiro, R. de Conti, N. Durán, Amazonian Biodiversity: Pigments from Aspergillus and Natural colorants production, Curr Trends Biotechnol Pharm 6 (2012) 300-311.

[11] V.C. Santos-Ebinuma, I.C. Roberto, M.F.S. Teixeira, A. Pessoa Jr, Improving of Red Colorants Production by a New Penicillium purpurogenum Strain in Submerged Culture and the Effect of Different Parameters in Their Stability, Biotechnol. Prog. 29 (2013) 778-785.

[12] L. Xijun, W. Changlu, G. Kunliang, Identification of new red pigments produced by Monascus rubber, Dyes Pigments 73 (2007) 121-125.

[13] L. Marie-Odile, M. Sandrine, Isolation and Structural Characterization of Two New Metabolites from Monascus, J. Agric. Food Chem. 58 (2010) 1800-1803.

[14] M. Dermiki, M.H. Gordon, P. Jauregi, Recovery of astaxanthin using colloidal gas aphrons (CGA): A mechanistic study, Sep. Purif. Technol. 65 (1) (2009) 5464.

[15] P.A.J. Rosa, I.F. Ferreira, A.M. Azevedo, M.R. Aires-Barros, Aqueous two-phase systems: a viable platform in the manufacturing of biopharmaceuticals, J. Chromatogr. A 1217 (2010) 2296-2305.

[16] V.C. Santos, F.A. Hasmann, A. Converti, A. Pessoa Jr., Liquid-liquid extraction by mixed micellar systems: A new approach for clavulanic acid recovery from fermented broth, Biochem. Eng. J. 56 (2011) 75-83.

[17] J.N. Israelachvili, D.J. Mitchell, B.W. Ninham, Theory of self-assembly of hydrocarbon amphiphiles into micelles and bilayers, J Chem Soc Far Trans II 72 (1976) 1525-1568 
[18] C.O. Rangel-Yagui, A. Pessoa Jr., L.C. Tavares, Micellar solubilization of drugs, ] Pharm Pharmaceut Sci 8 (2005) 147-163.

[19] D. Roy, R.R. Kommalapati, K.T. Valsaraj, W.D. Constant, Soil flushing of residual transmission fluid: application of colloidal gas aphron suspensions and conventional surfactant solutions, Water Res. 29 (1995) 589-595.

[20] J.F. Scamehorn, J.H. Harwell, Current trends and future developments in surfactant-based separations, in: J.F. Scamehorn, J.H. Harwell (Eds.), Surfactant Based Separations -Science and Technology, ACS Symposium series, Washington DC, 2000, pp. 1-14.

[21] P. Jauregi, G.R. Mitchell, J. Varley, Colloidal gas aphrons (cga): dispersion and structural features, AIChE J. 46 (1) (2000) 24-36.

[22] R.W. Alves, A.A.U. de Souza, S.M. de Arruda Guelli Ulson de Souza, P. Jauregi, Recovery of norbixin from a rawextraction solution of annatto pigments using colloidal gas aphrons (CGAs), Sep. Purif. Technol. 48 (2006) 208-213.

[23] A. Molaei, K.E. Waters, Aphron applications - A review of recent and current research, Adv. Colloid Interfac. 216 (2015) 36-54

[24] Z. Oleg, P. Vera, Influence of thermobaric conditions on size distribution of colloidal gas aphrons, Colloids and Surfaces A: Physicochem. Eng. Aspects. (2015), http://dx.doi.org/10.1016/j.colsurfa.2015.05.039 0927-7757.

[25] G. Spigno, P. Jauregi, Recovery of gallic acid with colloidal gas aphrons (CGA), Int. J. Food Eng. 1 (2005) 1-10.

[26] E. Fuda, P. Jauregi, An insight into the mechanism of protein separation by colloidal gas aphrons (CGA) generated from ionic surfactants, J. Chromatogr. B 843 (2006) 317-326.

[27] P. Jauregi, M. Dermiki, Separation of value-added bioproducts by colloidal gas aphrons (CGA) flotation and applications in the recovery of value-added food products, in: S.S.H. RIZVI (Ed.), Separation, Extraction and Concentration Processes in the Food, Beverage and Nutraceutical Industries, Woodhead Publishing, Ithaca, 2010, pp. 284-313 (Woodhead Publishing Series in Food Science, n.202).

[28] E. Fuda, D. Bhatia, D.L. Pyle, P. Jauregi, Selective separation of b-lactoglobulin from sweet whey using CGAs generated from a cationic surfactant CTAB, Biotechnol. Bioeng. 90 (2005) 532-542.

[29] M. Dermiki, M.H. Gordon, P. Jauregi, The use of Colloidal Gas Aphrons (CGA) as novel downstream processing for the recovery of astaxanthin from the cells of Phaffia rhodozyma, J. Chem. Technol. Biotechnol. 83 (2008) 174-182.

[30] S. Mukherjee, S. Mukhopadhyay, A. Pariatamby, M.A. Hashim, G. Redzwana, B. S. Gupta, Optimization of pulp fibre removal by flotation using colloidal gas aphrons generated from a natural surfactant, J. Taiwan Inst. Chem. Eng. 53 (2015) 15-21.
[31] J.I. Pitt, A laboratory guide to Common Penicillium species, CSIRO, Australia, 1985, p. 182.

[32] M.C. Amiri, E.T. Woodburn, A method for the characterization of colloidal gas aphrons, Trans. Inst. Chem. Eng. 68 (1990) 154-160.

[33] E.H.A. Mansur, Y. Wang Y. Dai, Removal of suspensions of fine particlesfrom water by colloidal gas aphrons (CGAs), Sep. Purif. Technol. 48 (2006) 71-77.

[34] N.E. Craft, J.H. Soares Jr., Relative solubility, stability, and absorptivity of lutein and $\beta$-carotene in organic solvents, J. Agric. Food Chem. 40 (1992) 431-434.

[35] OECD GUIDELINE FOR THE TESTING OF CHEMICALS. Adopted by the Council on 27th July 1995. Partition Coefficient (n-octanol/water): Shake Flask Method 107 Adopted: 27.07.95.

[36] S. Zhong, Z. Wang, Preparation and characterization of yellow Monascus pigments, Sep. Pur. Technol. 150 (2015) 139-144.

[37] M.R. Johns, D.M. Stuart, Production of pigments by Monascus purpureus in solid culture, J. Ind. Microbiol. 8 (1991) 23-38.

[38] V.C. Santos-Ebinuma, A.M. Lopes, A. Pessoa Jr., M.F.S. Teixeira, Extraction of natural red colorants from the fermented broth of Penicillium purpurogenum using aqueous two-phase polymer systems, Biotech. Progress 31 (2015) 1295 1304.

[39] M.J. Rosen, Foaming and antifoaming by aqueous solutions of surfactants, in: M.J. Rosen (Ed.), Surfactants and Interfacial Phenomena, John Wiley and Sons Inc, New Jersey, 2004, pp. 277-302.

[40] M. Mitschke, G.M. Pastore, Biossurfactantes: propriedade e aplicações, Quim. Nova 25 (2002) 772-776.

[41] M. Sarkar, S. Poddar, Studies on the Interaction of Surfactants with Cationic Dye by Absorption Spectroscopy. J. Colloid Interface Sci. 221 (2000) 181-185.

[42] M. Sarkar, S. Poddar, Spectral studies of methyl violet in aqueous solutions of different surfactants in supermicellar concentration region, Spectrochim. Acta A 55 (1999) 1737-1742.

[43] M.E. Diaz Garcia, A. Sanz-Medel, Dye-surfactant interactions: a review, Talanta 33 (1986) 255-264.

[44] S. Gokturk, Effect of hydrophobicity on micellar binding of carmínico acid, J Photoch. Photobio. A 169 (2005) 115-121.

[45] S. Gokturk, M. Tunçay, Spectral studies of safranin-O in different surfactant solutions, Spectrochim. Acta A-M 59 (2003) 1857-1866.

[46] S.P.M. Ventura, V.C. Santos-Ebinuma, J.F.B. Pereira, M.F.S. Teixeira, A. Pessoa, J A.P. Coutinho, Isolation of natural colorants from fermented broth using ionic liquid-based aqueous two-phase systems, J. Ind. Microbiol. Biotechnol. 40 (2013) 507-516.

[47] S.V. Save, V.G. Pangarkar, Characterisation of colloidal gas aphrons, Chem. Eng. Commun. 127 (1994) 35-54. 